\title{
Hereditary breast and ovarian cancer in Andalusian families: a genetic population study
}

\author{
Bella Pajares ${ }^{1 *}$, Javier Porta ${ }^{2}$, Jose María Porta ${ }^{2}$, Cristina Fernández-de Sousa ${ }^{1}$, Ignacio Moreno ${ }^{1}$, Daniel Porta ${ }^{2}$, \\ Gema Durán', Tamara Vega ${ }^{2}$, Inmaculada Ortiz², Carolina Muriel ${ }^{1}$, Emilio Alba and Antonia Márquez ${ }^{1}$
}

\begin{abstract}
Background: The BRCA1/2 mutation profile varies in Spain according to the geographical area studied. The mutational profile of BRCA1/2 in families at risk for hereditary breast and ovarian cancer has not so far been reported in Andalusia (southern Spain).
\end{abstract}

Methods: We analysed BRCA1/2 germline mutations in 562 high-risk cases with breast and/or ovarian cancer from Andalusian families from 2010 to 2015.

Results: Among the 562 cases, 120 (21.4\%) carried a germline pathogenic mutation in BRCA1/2; 50 in BRCA1 (41.7\%) and 70 in BRCA2 (58.3\%). We detected 67 distinct mutations (29 in BRCA1 and 38 in BRCA2), of which 3 in BRCA1 (c.845C > A, c.1222_1223delAC, c.2527delA) and 5 in BRCA2 (c.293 T > G, c.5558_5559delGT, c.6034delT, c.6650_6654delAAGAT, c.6652delG) had not been previously described. The most frequent mutations in BRCA1 were c.5078_5080delCTG (10\%) and C.5123C > A (10\%), and in BRCA2 they were c.9018C > A (14\%) and c.5720_5723delCTCT (8\%). We identified 5 variants of unknown significance (VUS), all in BRCA2 (c.5836 T > C, c.6323G > T, c.9501 + 3A > T, c.8022_8030delGATAATGGA, c.10186A > C). We detected 76 polymorphisms (31 in BRCA1, 45 in BRCA2) not associated with breast cancer risk.

Conclusions: This is the first study reporting the mutational profile of BRCA1/2 in Andalusia. We identified 21.4\% of patients harbouring BRCA1/2 mutations, 58.3\% of them in BRCA2. We also characterized the clinical data, mutational profile, VUS and haplotype profile.

Keywords: Hereditary breast and ovarian cancer, BRCA1/BRCA2 mutation, Genetic counselling, Recurrent mutation, Andalusian population

\section{Background}

About $5-10 \%$ of all breast cancer (BC) cases are due to inherited predisposition, and about $20-40 \%$ of these cases are caused by germline mutations in BRCA1 and BRCA2 genes $[1,2]$. Women with BRCA1/2 germline mutations have a high lifetime risk for developing both $\mathrm{BC}$ and ovarian cancer (OC) compared to women from the general population [3]. Both genes have a high allelic heterogeneity and more than 3500 DNA sequence

\footnotetext{
* Correspondence: bella.pajares@ibima.eu

${ }^{1}$ Clinical Oncology Unit Hospitales Universitarios Regional y Virgen de la Victoria. Instituto de Investigación Biomédica de Málaga (IBIMA), Campus Teatinos s/n. 29010, Malaga, Spain

Full list of author information is available at the end of the article
}

variants have been reported, including pathogenic mutations, polymorphisms and variants of unknown significance (VUS) [4]. Studies of polymorphisms and their haplotypes in BRCA1 and BRCA2 are necessary to establish the genetic structure of our population and their differences and similarities with other populations, as well as the possible relationship with the risk for $\mathrm{BC}$ or intrinsic subtypes. The prevalence and profile of BRCA1 and BRCA2 germline mutations show significant ethnic and geographic variation. In Spain, several studies have reported the mutational analysis of BRCA1/2 in families with hereditary breast and ovarian cancer (HBOC), noting considerable geographical variation regarding the prevalence of BRCA1 and BRCA2 pathogenic mutations,

(c) The Author(s). 2018 Open Access This article is distributed under the terms of the Creative Commons Attribution 4.0 International License (http://creativecommons.org/licenses/by/4.0/), which permits unrestricted use, distribution, and reproduction in any medium, provided you give appropriate credit to the original author(s) and the source, provide a link to the Creative Commons license, and indicate if changes were made. The Creative Commons Public Domain Dedication waiver (http://creativecommons.org/publicdomain/zero/1.0/) applies to the data made available in this article, unless otherwise stated. 
recurrent mutations, novel mutations and VUS (Fig. 1) [5]. This wide variations show that the multiple places of origin of Spanish families increases the variety of mutations in high risk HBOC spanish patients and modifies the frequency of recurrent mutations in each area. Although these studies cover many areas of Spain, none has yet been undertaken in southern Spain, the most populated region in our country and the closest to the Maghreb countries. Thus, no detailed information exists about HBOC, the mutational prevalence, the profile or polymorphisms/haplotypes of the BRCA1 and BRCA2 genes in Andalusia.

The aim of this study was to determine the mutational profile of BRCA1 and BRCA2 in 562 families at risk for HBOC from Malaga (recurrent mutations, novel mutations and VUS) and correlate the clinical characteristics of these patients with the mutational spectrum of BRCA1 and BRCA2. We also investigated genetic variants of these genes by studying BRCA1 and BRCA2 polymorphisms and haplotypes.

\section{Methods}

\section{Study population}

The study included a total of 562 index cases (ICs) of women at high risk for HBOC selected by the Family Cancer Unit at the Regional and the Virgen de la Victoria hospitals in Malaga, Spain, between 2010 and
2015. Families studied were unrelated, Spanish with a caucasian origin and with residence in Andalucia.

Genetic testing was offered to individuals from families at a high risk for $\mathrm{HBOC}$ meeting the 2011 criteria of the Spanish Society of Clinical Oncology (SEOM) [6] (Table 1). A total of 562 families fulfilled at least one of the selection criteria. The study was approved by the hospital ethics committee. All tested individuals provided signed informed consent following the appropriate genetic counselling. Genealogical trees were constructed on the basis of an index case considered to have the highest probability of being a deleterious mutation carrier (the male case or the youngest female case). None of the families met the strict criteria for other known syndromes involving BC such as Li-Fraumeni, ataxia telangiectasia or Cowden disease. Information concerning the status of oestrogen receptor (ER), progesterone receptor (PR) and HER2 protein was gathered from pathology and medical reports. The immunohistochemical technique was carried out in an automated immunostaining DAKO TechMate Horizon, using the EnVision system (DAKO) as a method for visualizing the antigen-antibody reaction. The antibodies used for staining were: Estrogen receptor (1D5, Dako); Progesterone receptor (PgR636, Dako) and c-erb-B2 (HERceptestTM, Dako). A luminal phenotype was considered when ER or

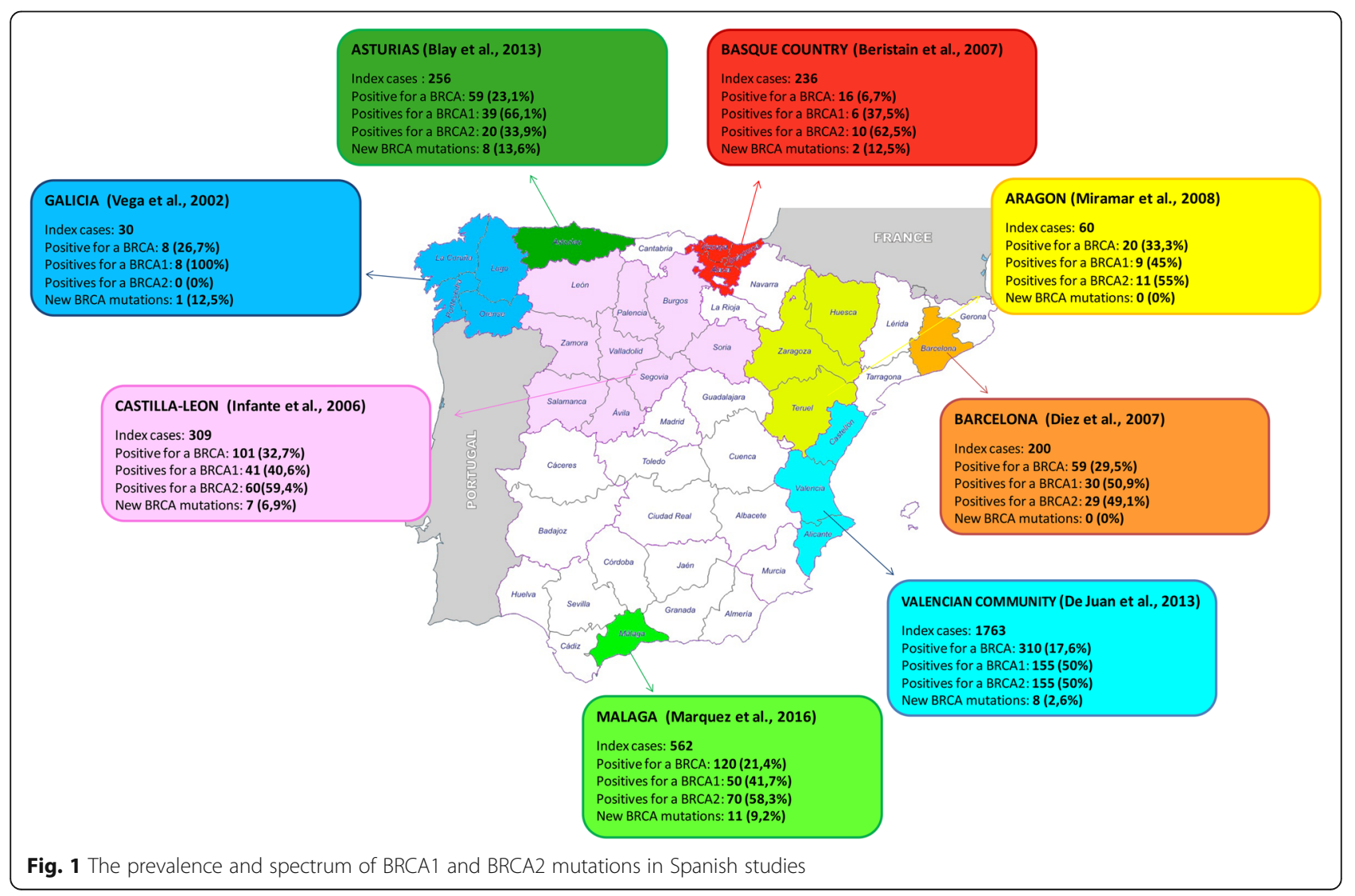


Table 1 Selection criteria

Families with three or more second degree relatives with breast cancer or ovarian cancer, at least two of which must be first degree.

1) Three or more family members with breast and/or ovarian cancer.

(Br: Only breast cancer. Ov: at least one ovarian cancer).

Families with two first degree relatives with breast cancer or ovarian cancer.

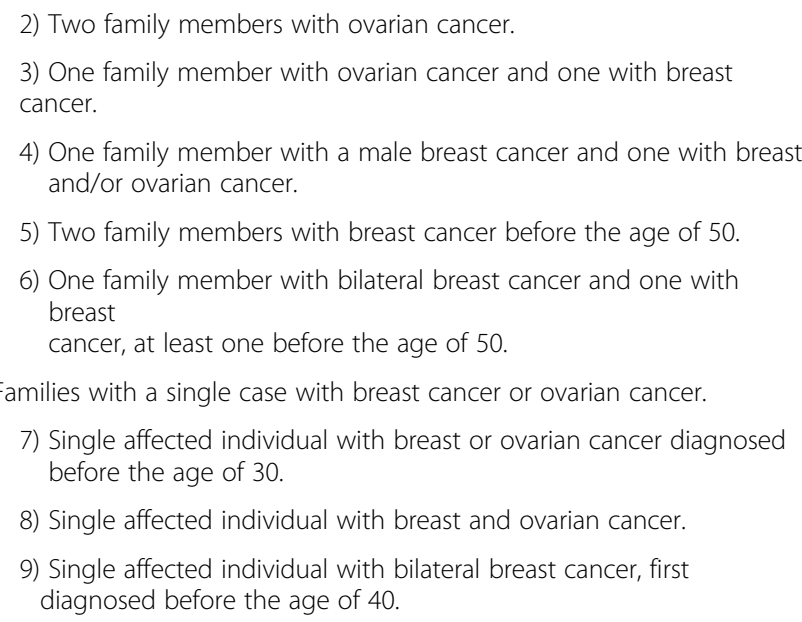

PR were positive by immunohistochemistry (IHC). A triple negative phenotype was considered if the tumours were ER, PR and HER2 negative and tumours were considered HER2 positive if the HER2 protein was positive by IHC $(+++)$ or by immunofluorescence (FISH/SISH), independently of the hormone receptor status.

\section{BRCA1 and BRCA2 mutation analysis}

Genomic DNA was obtained from blood using the DNeasy Blood \& Tissue Kit (QiaGen) according to the manufacturer's instructions. BRCA1 and BRCA2 coding regions and their intron-exon boundaries were amplified using PCR primers complementary to flanking intron sequences. Primers were designed by primer 3 software [7] and then evaluated by single nucleotide polymorphism (SNP) check software [8] to test for the presence of SNPs in their length, especially at the 3' end. Sequencing reactions were performed by using an ABI Prism Big Dye Terminator v3.1 Cycle Sequencing Kit (Applied Biosystems). Sequenced PCR products were purified using CentriSeptfiltration columns (Applied Biosystems) following the manufacturer's instructions. Sequencing was carried out using an ABI 3130 genetic analyser (Applied Biosystems). Visual inspection of base calling was used to evaluate the quality of DNA sequencing. NCBI reference sequences (RefSeq) NM_007294.3 and NM 000059.3 were used for the annotation of BRCA1 and BRCA2 variants, respectively. These RefSeq transcripts are included in the Locus Reference Genomic (LRG) data LRG_292-BRCA1 and LRG_293-BRCA2. Bi-directional sequencing review was performed using
Mutation Surveyor Software (v.5.0.0, Soft Genetics, State College, PA). BRCA1/2 variant data were submitted to the Clinical Variation Database (ClinVar) [9].

\section{Large genomic rearrangements in BRCA1 and BRCA2}

Screening for large genomic rearrangements (LGRs) in BRCA1 and BRCA2 was performed by multiplex ligation-dependent probe amplification (MLPA) using SALSA MLPA probemix P002 BRCA1 and SALSA MLPA probemix P090 BRCA2 kits according to the manufacturer's instructions (MRC-Holland). MLPA products were analyzed using Genetic Analyzer ABI 3130 (Applied Biosystems). MLPA fragment analysis and comparative analysis were performed using Coffalyser. Net software (MRC-Holland) using 8 control samples to set up for peak height normalization and reaction quality control calculations.

\section{Mutation nomenclature and classification}

The nomenclature of the sequence variants identified followed the guidelines of the Human Genome Variation Society (HGVS) recommendations, version 15.11 [10]. The recommendation of the American College of Medical Genetics and Genomics (ACMG) and the Association for Molecular Pathology (AMP) were followed to standardize interpretation and reporting of genomic results [11]. Five publicly accessible BRCA1 and BRCA2 variant databases were consulted for clinical classification of variants: ClinVar [9], Universal Mutation Database (UMD) [12], Breast Cancer Mutation Data Base [13], Human Gene Mutation Database (HGMD) [14] and Leiden Open Variation Database (LOVD) [15], as well as the associated bibliography. In silico analysis of the VUS identified was performed using available software, such as PolyPhen 2 [16], PANTHER [17], PhD-SNP [18], SNAP [19], Meta-SNP [20] or SIFT [21] and four different splice-site prediction algorithms: Human Splicing Finder [22], Gene Splicer [23], Splice Site Prediction [24] and MaxEntScan [25]. To predict the functional effect of indels we used PROVEAN Genome Variants software [26].

For the classification of novel mutations we have followed the criteria of the ACMG [11] (American College of Medical Genetics and Genomics). Specifically, the 8 novel mutations considered pathogenic met the following criteria: Pathogenic very strong criterion (PVS1) null variant (2 nonsense and 6 frameshift) in a gene where the loss of function (LOF) is a known mechanism of disease. Moderate criterion of evidence for pathogenicity (PM2); Absent from controls in Exome Sequencing Project, 1000 Genomes Project, or Exome Aggregation Consortium.

\section{Statistics}

Statistical analysis was done using SPSS v.11 software. The estimation of mutation carrier probabilities in 
BRCA1 or BRCA2 genes for different individuals (tumour phenotypes, other tumours) or familial phenotypes (inclusion criteria, other tumours) was computed by conditional logistic regression with covariates (step by step; backwards Wald). Inclusion criteria were introduced as independent variables and mutation types as dependent variables. To compare two proportions we used the Z-test. The non-parametric Man-Whitney U or Kruskal-Wallis tests were used for comparison of two or more independent quantitative variables. The level of significance considered in all tests was $5 \%$.

\section{Population study}

Genetic variants were classified as either deleterious mutations or common genetic variants. Variants tagged as common polymorphisms were selected to make a genetic population study based on haplotype frequencies. Of all the SNPs obtained in the BRCA1 sequence during clinical testing we selected 14 SNPs (IVS4-49C/T, IVS8-58delT, Q356R, D693N, S694S, L771 L, P871L, E1038G, S1040 N, K1183R, R1347G, S1436S, S1613G, M1652I) that had previously been used to establish 10 canonical haplotypes [27] and in the BRCA2 sequence we selected the six most frequent polymorphisms (rs144848, rs1801406, rs543304, rs1799955, rs9534262, rs11571818). Haplotype pairs based on BRCA1 and BRCA2 genotypes were generated using the software DnaSP 3.00 [28]. Comparison of allele frequencies was performed using the $\mathrm{X}^{2}$ test or Fisher exact test when necessary. The strength of the association between different categories was stated using the OR and its 95\% confidence intervals calculated by the exact method. The associations between BRCA1 and BRCA2 SNPs, haplotypes, BC risk, and molecular subtypes were analysed using logistic regression.

\section{Results}

\section{General characterisation}

A cohort of 562 index cases was analysed during 20102015 (following SEOM 2011 criteria. Table 1). Among the 562 index cases, 295 (52.5\%) had one inclusion criterion for a high for HBOC, 157 (27.9\%) had two criteria and $110(19.6 \%)$ had three or more criteria. The most frequent criterion was "Three or more family members with breast and/or ovarian cancer" (317 cases, 56.4\%), followed by "two second/first degree relatives with breast cancer under 50" (284 cases, 50.5\%). The probability of harbouring BRCA mutations increased with the number of HBOC criteria. Of the 120 BRCA-mutated families, 94 (78\%) met more than 1 criterion for $\mathrm{HBOC}$, whereas of the 441 wild type (wt) families only 172 (39\%) met more than 1 criterion for HBOC $(p<0.0001)$.

The vast majority of patients (559) had $\mathrm{BC}$ as the primary tumour (99.5\%) and 3 had a history of OC $(0.5 \%)$.
These OC patients were included according to selection criteria number 2 ("two family members with ovarian cancer"). Among the BC patients, 495 were under the age of $50(88.1 \%)$, bilateral BC was observed in 119 cases (21.2\%), and 21 families (3.7\%) had a male BC history. Among the 562 index cases, 356 (63.3\%) had other primary tumours apart from the breast or ovarian cancer related to the syndrome studied. Colorectal cancer (CRC) was the most frequent tumour $(n=111,19.8 \%)$, followed by prostate cancer $(n=86,15.3 \%)$ and lung cancer $(n=69,12.3 \%)$ (Additional file 1: Table S1).

Among the BRCA1-mutated families, the most frequent tumours were $\mathrm{CRC}$, lung, gastric, and head and neck cancer (16, 14, 12 and 12\%, respectively). Among the BRCA2-mutated families, the most frequent tumours were prostate, lung, CRC and gastric cancer (24.3, 15.7, 14.3 and $8.6 \%$, respectively).

The presence of prostate cancer was significantly higher in BRCA2 compared to BRCA1 patients or wild type patients $(p<0.05)$. The most frequent second primary tumours according to BRCA status are shown in Additional file 2: Table S2.

Luminal was the most frequent $\mathrm{BC}$ tumour phenotype. The frequency of $\mathrm{BC}$ tumour phenotypes is shown in Additional file 3: Table S3. Among the BRCA1 patients, triple negative (TNBC) was the most frequent phenotype (44\%), and among the BRCA2 patients, luminal was the most frequent phenotype (64.3\%). One case of pathogenic mutation in BRCA1 and another in BRCA2 had a HER2-positive phenotype (2 and 1.4\%, respectively).

\section{Mutational spectrum}

Among the 562 index cases, 120 (21.4\%) carried a germline pathogenic mutation in BRCA1/2 genes (Fig. 1, Additional file 4: Table S4 and Additional file 5: Table S5). Among the 120 positive cases, we identified 50 with BRCA1 pathogenic mutations (41.7\%) and 70 with BRCA2 pathogenic mutations (58.3\%).

Analysis of the BRCA1 gene revealed 29 distinct germline mutations. Among these 29 mutations, 3 (10.3\%) are novel mutations not described so far. The most prevalent mutations were frame-shift deletions $(N=19 ; 38 \%)$, missense $(N=10 ; 20 \%)$, nonsense $(N=7$; $14 \%)$, in-frame deletions $(N=5 ; 10 \%)$, frame-shift insertion $(N=4 ; 8 \%)$, large rearrangements $(N=3 ; 6 \%)$ and mutations in the intervening splicing sequence $(N=2 ; 4 \%)$ (Fig. 2a). We identified 3 novel frame-shift mutations that are not listed in the conventional databases (BIC, UMD, HGMD and ClinVar databases) and, as far as we know, have not been published (Table 2).

Analysis of the BRCA2 gene revealed 38 distinct germline mutations. Among these 38 mutations, 5 (13.2\%) are novel frame-shift mutations not previously described in the conventional databases (Table 2). One of the novel 


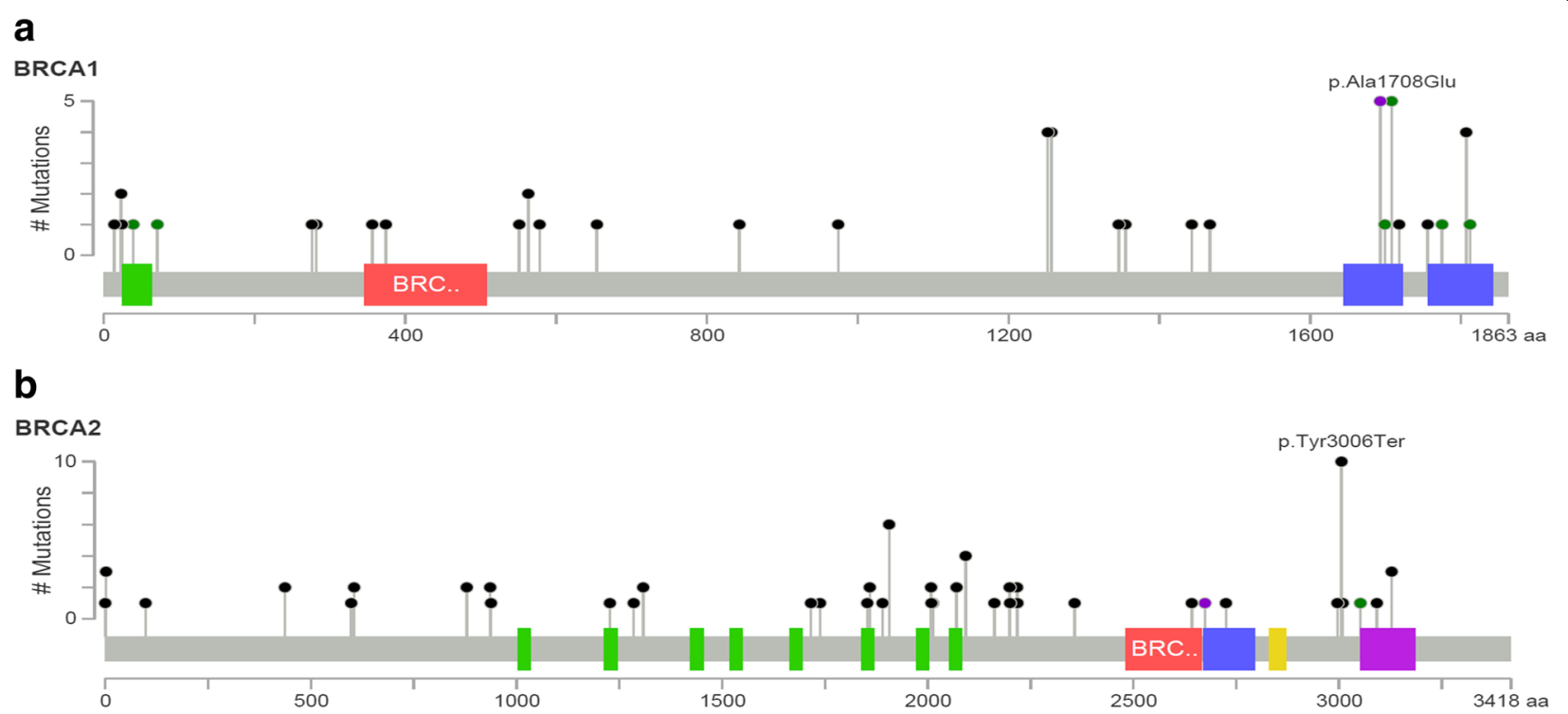

Fig. 2 Distribution and mutational profile along BRCA 1 and BRCA2 genes Lollipop plot showing the distribution and mutation profile in a BRCA1 and $\mathbf{b}$ BRCA2. The truncation variants (nonsense, frameshift indels) are shown in black, missense type in green and the others in purple. The large deletions are not shown in the figure. On the vertical axis we show the frequency of appearance of each mutation. On the horizontal axis we show the aminoacid position of each mutation

mutations identified in BRCA2 was present in two apparently unrelated families. The most prevalent mutations were frame-shift $(N=48 ; 68.6 \%)$, nonsense $(N=16$; $22.9 \%)$, in-frame deletions $(\mathrm{N}=1 ; 1.43 \%)$, mutations in the intervening splicing sequence $(\mathrm{N}=4 ; 5.71 \%)$ and missense $(\mathrm{N}=1 ; 1.43 \%)$ (Fig. 2b). We detected the same large deletion of BRCA1 exons 1-13 in three unrelated families (6\%) and no deletions and/or insertions in BRCA2.

\section{Recurrent mutations}

The 5 most recurrent mutations in BRCA1 and 5 most recurrent in BRCA2 identified in our population represent 44 and $40 \%$ of the mutations found in these genes, respectively (Tables 3 and 4). The most frequent mutations observed in BRCA1 were c.5078_5080delCTG (p.Ala1693del) and c.5123C > A (p.Ala1708Glu), followed by c.3756_3759delGTCT, c.3770_3771delAG and c.5419delA. The most recurrent BRCA2 variant in our study was c.9018C > A (p.Tyr3006Ter), identified in 10 of the 70 positive BRCA2 families (14.3\%). This nonsense variant results in a premature stop codon and is predicted to encode a truncated non-functional protein. The next most recurrent BRCA2 variant, c.5720_5723delCTCT, is caused by a deletion, producing a shift in the translational

Table 2 Novel pathogenic mutations not described in databases

\begin{tabular}{|c|c|c|c|c|c|c|c|c|c|c|c|}
\hline Gene & Exon & $\begin{array}{l}\text { HGVS CDNA } \\
\text { Based Designation }\end{array}$ & $\begin{array}{l}\text { HGVS Protein } \\
\text { Based Designation }\end{array}$ & $\begin{array}{l}\text { Mutation } \\
\text { Type }\end{array}$ & Criteria & $\begin{array}{l}\text { Breast } \\
\text { Cancer (BC) }\end{array}$ & $\begin{array}{l}\mathrm{BC}< \\
50\end{array}$ & $\begin{array}{l}\text { Bilateral } \\
\text { BC }\end{array}$ & $\begin{array}{l}\text { Ovarian } \\
\text { Cancer }\end{array}$ & $\begin{array}{l}\text { Other } \\
\text { tumours }\end{array}$ & Phenotype \\
\hline BRCA1 & 11 & $c .845 C>A$ & p.Ser282Ter & NS & $1,2,8$ & YES & YES & $\mathrm{NO}$ & YES & & Triple-negative \\
\hline BRCA1 & 11 & c.1222_1223delAC & p.Thr374Ter & FSD & $1,2,7$ & YES & YES & $\mathrm{NO}$ & YES & & Unknown \\
\hline BRCA1 & 11 & c.2527delA & p.Thr843GInfs*3 & FSD & 1,5 & YES & YES & $\mathrm{NO}$ & $\mathrm{NO}$ & Colon, lung & Luminal \\
\hline BRCA2 & 7 & c.293 T> G & p.Leu98Ter & NS & $1,5,7$ & YES & YES & YES & $\mathrm{NO}$ & & Luminal \\
\hline BRCA2 & 11 & c.5558_5559delGT & p.Cys1853Cysfs*4 & FSD & $1,5,7$ & YES & YES & YES & $\mathrm{NO}$ & Prostate & Luminal \\
\hline BRCA2 & 11 & c.6034delT & p.Ser2012Profs*28 & FSD & 1,5 & YES & YES & $\mathrm{NO}$ & $\mathrm{NO}$ & & Unknown \\
\hline BRCA2 & 11 & $\begin{array}{l}\text { c.6650_6654delAAGA } \\
T\end{array}$ & p.Lys2217llefs*6 & FSD & $1,5,7$ & YES & YES & YES & YES & & Luminal \\
\hline BRCA2 & 11 & $\begin{array}{l}\text { c.6650_6654delAAGA } \\
T\end{array}$ & p.Lys2217llefs*6 & FSD & $1,3,7$ & YES & YES & $\mathrm{NO}$ & YES & Lung & Luminal \\
\hline BRCA2 & 11 & c.6652delG & p.Asp2218llefs*11 & FSD & $1,5,6$ & YES & YES & YES & NO & Prostate & Luminal \\
\hline
\end{tabular}


Table 3 Recurrent mutations of BRCA1 gene identified in this study

\begin{tabular}{|c|c|c|c|c|c|c|c|c|c|}
\hline $\begin{array}{l}\text { Recurrent mutations } \\
\text { in BRCA1 }\end{array}$ & Family & Criteria & Breast Cancer (BC) & Male BC & $\mathrm{BC}<50$ & Bilateral BC & $\begin{array}{l}\text { Ovarian } \\
\text { Cancer }\end{array}$ & Other tumours & Phenotype \\
\hline \multirow{5}{*}{$\begin{array}{l}\text { 5078_5080delCTG } \\
\text { (p.Ala1693del) }\end{array}$} & 40 & $1,2,8$ & Yes & No & Yes & Yes & Yes & ENT & Unknown \\
\hline & 252 & $1,5,6$ & Yes & No & Yes & Yes & No & $\begin{array}{l}\text { Lung, endometrial } \\
\text { and kidney }\end{array}$ & Triple negative \\
\hline & 525 & 1,6 & Yes & No & Yes & Yes & No & & Luminal \\
\hline & 537 & $2,7,8$ & Yes & No & Yes & No & Yes & & Unknown \\
\hline & 669 & $1,2,8$ & Yes & No & Yes & No & Yes & & Luminal \\
\hline \multirow[t]{5}{*}{$\begin{array}{l}\text { c.5123C > A } \\
\text { (p.Ala1708Glu) }\end{array}$} & 207 & 1,6 & Yes & No & Yes & Yes & No & & $\begin{array}{l}\text { Triple negative - } \\
\text { Luminal }\end{array}$ \\
\hline & 507 & $1,2,8$ & Yes & No & Yes & No & Yes & ENT & Unknown \\
\hline & 811 & $1,3,5$ & Yes & No & Yes & No & Yes & & Luminal \\
\hline & 836 & $1,5,6$ & Yes & No & Yes & Yes & No & & Triple negative \\
\hline & 861 & 1,5 & Yes & No & Yes & No & No & Endometrial, gastric & $\begin{array}{l}\text { Triple negative - } \\
\text { Luminal }\end{array}$ \\
\hline \multirow{4}{*}{$\begin{array}{l}\text { c.3756_3759delGTCT } \\
\text { (p.Ser1253Argfs) }\end{array}$} & 253 & 5 & Yes & No & Yes & No & No & & Unknown \\
\hline & 337 & $1,7,8$ & Yes & No & Yes & No & Yes & $\begin{array}{l}\text { Lung, colon and } \\
\text { multiple myeloma }\end{array}$ & Triple negative \\
\hline & 823 & 1,5 & Yes & No & Yes & No & No & & Luminal - HER2 \\
\hline & 1070 & $1,5,8$ & Yes & No & Yes & No & Yes & $\begin{array}{l}\text { Cerebral and germinal } \\
\text { tumour }\end{array}$ & Unknown \\
\hline \multirow{4}{*}{$\begin{array}{l}\text { c.3770_3771delAG } \\
\text { (p.Glu1257Glyfs) }\end{array}$} & 7 & 1,5 & Yes & No & Yes & Yes & No & Skin & Triple negative \\
\hline & 62 & $1,3,6$ & Yes & No & Yes & No & Yes & Colon & Triple negative \\
\hline & 859 & $1,3,5$ & Yes & No & Yes & No & Yes & $\begin{array}{l}\text { Colon, kidney and } \\
\text { bladder }\end{array}$ & Triple negative \\
\hline & 984 & $1,3,6$ & Yes & No & Yes & Yes & Yes & ENT and lymphoma & Luminal \\
\hline \multirow{4}{*}{$\begin{array}{l}\text { c.5419delA } \\
\text { (p.lle1807Leufs) }\end{array}$} & 481 & 1,5 & Yes & No & Yes & No & No & & Unknown \\
\hline & 690 & $1,3,5$ & Yes & No & Yes & No & Yes & & Triple negative \\
\hline & 835 & $1,3,6$ & Yes & No & Yes & Yes & Yes & Colon and lung & Triple negative \\
\hline & 983 & 3,5 & Yes & No & Yes & No & Yes & Colon & Triple negative \\
\hline
\end{tabular}

reading frame and leading to a premature stop codon (p.Ser1907Terfs). No genotype-phenotype correlation was observed regarding recurrent mutations.

\section{Novel mutations}

Eight new mutations were identified (not described in the BIC, UMD, HGMD or ClinVar databases). Three in BRCA1 (c.845C > A, c.1222_1223delAC, c.2527delA) and five in BRCA2 (c.293 T> G, c.5558_5559delGT, c.6034delT, c.6650_6654delAAGAT, c.6652delG) had not been previously described. Six of the new mutations identified are frameshift alterations and lead to the formation of an altered and probably non-functional protein. The other two are nonsense mutations that result in a premature stop codon. One of these novel BRCA2 mutations (p.Lys2217IlefsX6) was shared by 2 apparently unrelated families. The clinical and pathological characteristics of these families are shown in Table 2.
Variants of unknown significance (VUS)

In our study we found 5 VUS, all of them in BRCA2 (Table 5). These VUS are described below. No VUS in coexistence with the pathogenic variants were detected in this study.

\section{BRCA2: C.5836 T>C (p.Ser1946Pro)}

The variant c.5836 $\mathrm{T}>\mathrm{C}$ in BRCA2 results in the change of a Serine to a Proline (p.Ser1946Pro). This variant is also defined as $6064 \mathrm{~T}>\mathrm{C}$ using alternate nomenclature. The BRCA2 Ser1946Pro mutation was not observed at a significant allele frequency in 1000 genomes. Since serine and proline differ in polarity, charge, size and other properties, this is considered a non-conservative amino acid substitution. BRCA2 Ser1946Pro occurs at a position neither conserved nor located in a known functional domain. In silico analyses predict that this variant is unlikely to alter protein structure or function. Based 
Table 4 Recurrent mutations of BRCA2 gene identified in this study

\begin{tabular}{|c|c|c|c|c|c|c|c|c|c|}
\hline $\begin{array}{l}\text { Recurrent mutations } \\
\text { in BRCA1 }\end{array}$ & Family & Criteria & Breast Cancer (BC) & Male BC & $\mathrm{BC}<50$ & Bilateral BC & Ovarian Cancer & Other tumours & Phenotype \\
\hline \multirow{10}{*}{$\begin{array}{l}\text { c.9018C > A } \\
\text { (p.Tyr3006Ter) }\end{array}$} & 94 & $1,6,7$ & Yes & No & Yes & Yes & No & Lung & Luminal \\
\hline & 134 & $1,2,9$ & Yes & No & Yes & Yes & Yes & Melanoma & Luminal \\
\hline & 264 & $1,5,7$ & Yes & No & Yes & No & Yes & Colon and gastric & Luminal \\
\hline & 265 & $1,5,7$ & Yes & No & Yes & No & No & Lung & Luminal \\
\hline & 274 & $1,5,7$ & Yes & No & Yes & No & No & Lung & Luminal \\
\hline & 787 & 1,6 & Yes & No & Yes & Yes & No & $\begin{array}{l}\text { ENT, pancreatic } \\
\text { and lung }\end{array}$ & Luminal \\
\hline & 831 & 1 & Yes & No & Yes & No & No & Colon & Triple negative \\
\hline & 1045 & 1,6 & Yes & No & Yes & Yes & No & $\begin{array}{l}\text { Gastric, pancreas } \\
\text { and prostate }\end{array}$ & Luminal \\
\hline & 1056 & $1,2,4$ & Yes & Yes & Yes & No & Yes & Colon & Luminal \\
\hline & 1063 & 5 & Yes & No & Yes & No & No & & Luminal \\
\hline \multirow{6}{*}{$\begin{array}{l}\text { c.5720_5723delCTCT } \\
\text { (p.Ser1907Terfs) }\end{array}$} & 84 & 1 & Yes & No & No & No & No & Thyroid & Unknown \\
\hline & 217 & $1,4,5$ & Yes & Yes & Yes & No & Yes & Lung and lymphoma & $\begin{array}{l}\text { Triple negative - } \\
\text { Luminal }\end{array}$ \\
\hline & 284 & $4,5,6$ & Yes & Yes & Yes & Yes & No & Germinal & Luminal \\
\hline & 286 & $1,4,5$ & Yes & Yes & Yes & No & No & & Luminal \\
\hline & 824 & 5 & Yes & No & Yes & No & No & Prostate & Luminal \\
\hline & 960 & $1,3,5$ & Yes & No & Yes & No & No & Germinal & Unknown \\
\hline \multirow{4}{*}{$\begin{array}{l}\text { c.6275_6276delTT } \\
\text { (p.Leu2092Profs) }\end{array}$} & 149 & 1,5 & Yes & No & Yes & No & No & Prostate & Luminal \\
\hline & 263 & 1,5 & Yes & No & Yes & No & No & Prostate & Luminal \\
\hline & 781 & $1,3,5$ & Yes & No & Yes & No & Yes & & Luminal \\
\hline & 925 & 5,6 & Yes & No & Yes & Yes & No & Bile duct & Luminal \\
\hline \multirow{4}{*}{$\begin{array}{l}\text { c.9382C > T } \\
\text { (p.Arg3128Ter) }\end{array}$} & 77 & $1,5,6$ & Yes & No & Yes & Yes & No & ENT & Luminal \\
\hline & 291 & $1,5,7$ & Yes & No & Yes & No & No & & Luminal \\
\hline & 329 & $1,4,5$ & Yes & Yes & Yes & Yes & No & ENT & Unknown \\
\hline & $?$ & $1,4,5$ & Yes & No & Yes & No & No & & Luminal \\
\hline \multirow[t]{3}{*}{$c .67+2 T>C$} & 750 & $5,7,8$ & Yes & No & Yes & No & Yes & ENT & Luminal \\
\hline & 905 & 6 & Yes & No & Yes & Yes & No & $\begin{array}{l}\text { Gastric, bladder } \\
\text { and melanoma }\end{array}$ & Luminal \\
\hline & 1051 & 5 & Yes & No & Yes & No & No & $\begin{array}{l}\text { Prostate, gastric } \\
\text { and colon }\end{array}$ & Luminal \\
\hline
\end{tabular}

on currently available evidence, it is unclear whether BRCA2 Ser1946Pro is a pathogenic or benign variant.

\section{BRCA2: C.6323G > T (p.Arg2108Leu)}

This sequence change has been reported in individuals in the Breast Cancer Information Core database [29] and the UMD [30], but has not been reported in the literature and is not present in population databases. In the UMD, this variant coexists with a pathogenic allele identified in the BRCA1 gene, which suggests that this c.6323G > T substitution in BRCA2 was not the primary cause of disease in that individual. In silico analyses

Table 5 BRCA2 variants of unknown significance (VUS)

\begin{tabular}{lllll}
\hline Gene & c.DNA (HGVS) & Prot. (HGVS) & Variant Type & Clinical data \\
\hline BRCA2 & c.5836 T $>$ C & p.Ser1946Pro & Misssense & Two cases of Breast Cancer (BC), one bilateral before 40 \\
BRCA2 & c.6323G $>$ T & p.Arg2108Leu & Misssense & One case of BC before 30 and three cases of prostate cancer \\
BRCA2 & c.9501 + 3A $>$ T & IVS25 +3A $>$ T & Splice site & One case of Triple negative BC before 40 \\
BRCA2 & c.8022_8030delGATAATGGA & p.Lys2674Lysdel & IFD & Two cases of BC before 50 \\
BRCA2 & c.10186A $>C$ & p.Ser3396Arg & Misssense & Two cases of BC before 50 \\
\hline
\end{tabular}


predict that this variant is unlikely to alter protein structure or function but these predictions have not been confirmed by published functional studies.

\section{BRCA2: $C .9501+3 A>T$ (IVS25 + 3A>T)}

This variant consists of an $\mathrm{A}>\mathrm{T}$ nucleotide substitution at the +3 position of intron 25 of the BRCA2 gene. This variant has been observed in several breast and/or ovarian cancer families [31-33]. In vitro and in vivo RNA studies report that BRCA2 c.9501 + 3A > T results in skipping of exon 25 [33-35]. The Splicing Working Group of the Evidence-Based Network for the Interpretation of Germline Mutant Alleles (ENIGMA) concluded that BRCA2 c. $9501+3 \mathrm{~A}>\mathrm{T}$ produces unequivocal splicing aberrations [36]. However, a large in vitro minigene splicing assay quantified the aberrant splicing and found that this variant results in less than $15 \%$ aberrant transcript, meaning that the full length transcript is predominant [37].

\section{BRCA2: C.8022_8030delGATAATGGA (p.Lys2674Lysdel)}

This variant has not been reported in the literature and is not described in variation databases (ClinVar, LOVD, InSight, UMD). This nine-nucleotide deletion in BRCA2 gives rise to a three-amino acid deletion (IME) in the putative gene product. In silico analysis by PROVEAN software predicted deleterious effects in the protein structure or function but these predictions have not been confirmed by published functional studies. This variant has been shown to co-segregate with disease in this family, but we have to consider that co-segregation of a sequence variant does not prove that the variant is causative. Based on currently available evidence, it is unclear whether BRCA2 c.8022_8030delGATAATGGA is a pathogenic or benign variant, and more studies are required to classify this variant.

\section{BRCA2: C.10186A > C (p.Ser3396Arg)}

This sequence change replaces serine with arginine at codon 3396 of the BRCA2 protein (p.Ser3396Arg). The serine residue is weakly conserved and there is a considerable physicochemical difference between both aminoacids. This variant is not present in population databases (ExAC without frequency) or variant databases (ClinVar, LOVD, InSight, UMD) and has not been reported in the literature in individuals with a BRCA2-related disease. Algorithms developed to predict the effect of missense changes in protein structure and function (SIFT, PolyPhen-2, Align-GVGD) suggest that this variant is tolerable, but these predictions have not been confirmed by published functional studies. In short, this is a new missense variant that is not expected to affect the function of the protein or cause disease. However, the evidence is insufficient at this time to state this conclusively.

\section{Polymorphisms and haplotypes}

Study of genetic variants detected during clinical BRCA1 and BRCA2 mutation screening of 562 patients by direct DNA sequencing showed 76 polymorphisms (31 in BRCA1 and 45 in BRCA2). The genotype frequencies among unrelated carriers were consistent with the expected frequencies under the assumption of Hardy-Weinberg equilibrium. In general, there were no significant differences between the allele frequencies of these polymorphisms and those obtained from Exac.

Of the polymorphisms obtained in the BRCA1 sequence we selected 14 for the assignment of haplotype pairs to the patient samples (IVS4-49C/T, IVS8-58delT, Q356R, D693N, S694S, L771 L, P871L, E1038G, S1040 N, K1183R, R1347G, S1436S, S1613G, M1652I), as previously established by Judkins et al. [27], resulting in 10 canonical haplotypes (Fig. 3a). From the 6 polymorphisms selected in BRCA2 we obtained 9 canonical haplotypes. The results of the BRCA2 haplotype frequencies are represented by a cladogram in Fig. $3 \mathrm{~b}$.

No differences were found in the haplotype frequencies between BRCA mutation carriers and non carriers, nor was there any evidence of an association between the genotypes, haplotypes and $\mathrm{BC}$ risk. Furthermore, no significant associations emerged between individual SNPs and molecular subtypes.

\section{Discussion}

This is the first study analysing BRCA1/BRCA2 germline mutation frequencies in Andalusia (southern Spain), the second largest autonomous community in Spain and the closest to the Maghreb. Though our results come from a single institution, the Family Cancer Unit of Malaga (Spain), they nevertheless concern a large cohort of patients (562 index cases) selected using the homogeneous criteria recommended by SEOM 2011. Regarding clinical data, the most frequent criterion in our cohort was "Three or more family members with breast and/or ovarian cancer" (317 cases, 56.4\%), as observed in other Spanish studies [38-40]. We also found a high frequency of melanoma, prostate cancer and male $\mathrm{BC}$ in BRCA2-mutated families, concordant with published literature [41]. No male BC was detected in BRCA1-mutated families. TNBC was the most frequent phenotype in BRCA1-mutated patients and luminal in BRCA2, concordant with published studies [42, 43]. Notably, we found the unusual circumstance of one case of a pathogenic mutation in BRCA1 and another in BRCA2 presenting a HER2-positive phenotype, a molecular subtype not frequently associated with BRCA deficiency. Their characterisation by molecular features was not possible because the tumour tissue was not available. 

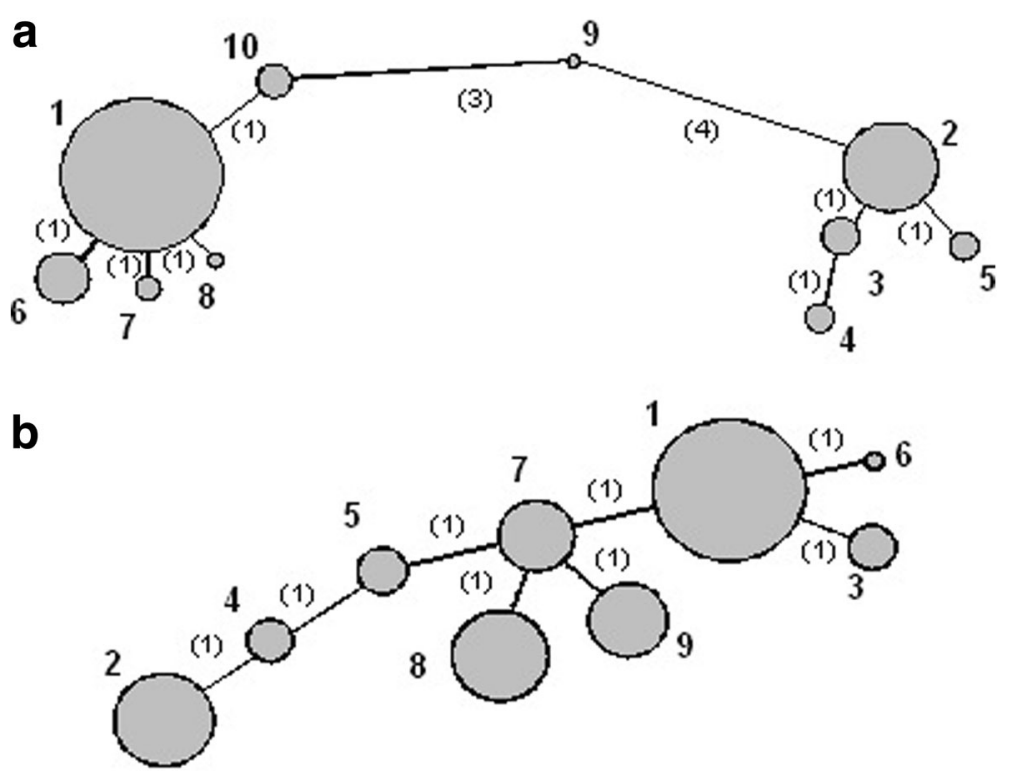

Fig. 3 Cladogram from BRCA1 and BRCA2 haplotypes. a Phylogenetic tree for the ten canonical BRCA1 haplotypes. b Phylogenetic tree for the ten canonical BRCA2 haplotypes. Circles represent different haplotypes with the diameters being proportional to the prevalence in this study. The distance between each circle is inversely proportional to genetic relatedness between haplotypes. The numbers in parentheses indicate how many of the polymorphisms differed between haplotypes

Among the 562 index cases $120(21.4 \%)$ carried a germline pathogenic mutation in BRCA1/2 genes. The rate of mutations in Spanish families at high risk for HBOC varies from 7 to $33 \%$ [38, 40, 44]. Our mutation rate $(21.4 \%)$ is comparable to previous studies published between 2010 and 2015 with similar inclusion criteria $(23 \%)[39,44]$, but different from studies prior to 2010 (27-34\%), probably due to the use of more restrictive selection criteria $[39,45,46]$. Among 120 positive cases, we identified 50 patients with BRCA1 mutations (41.7\%) and 70 with BRCA2 mutations (58.3\%). The prevalence and spectrum of BRCA1 and BRCA2 mutations in our population differed slightly from other Spanish studies (Fig. 1). The higher incidence of BRCA2 mutations in our study has also been reported in Extremadura [47], the Basque country [38], Castilla-León [48] and Aragon [40] but not in other regions such as Asturias [44] or Galicia [49], where the rate of BRCA1 mutations is higher than the rate of BRCA2 mutations. In Valencia BRCA1/BRCA2 ratios are similar [39]. These results are probably due to differences in the genetic background of the study population and not so much to the selection criteria or the analytical methods used.

Our study population shows clear influences from other national populations. The BRCA1 mutation c.211A > G (p.Arg71Gly), with a founding origin in Galicia and reported in 11 Spanish families [45, 50], was found once in our study. We also identified the BRCA2 frameshift mutation 5374delTATG, a highly prevalent mutation in Castilla-León (Spain). Regarding non-Spanish populations, the most common mutations in BRCA1(185delAG and 5382insC), reported in several international studies and frequent in Ashkenazi Jews (0.9 and 0.1\%, respectively), was not found in our study $[51,52]$. The low prevalence of both mutations in our country could be due to the absence of population mixing or large-scale migration from these areas to the Iberian Peninsula. In our study, no large rearrangements in BRCA2 were identified, which agrees with the results of others describing a higher rate of reordering in BRCA1 than in BRCA2 [53]. Regarding BRCA1, we detected the same large deletion of BRCA1 exons 1-13 in three unrelated families (6\%).

Regarding recurrent mutations, the most frequent mutation observed in BRCA1 was c.5078_5080delCTG, not reported as recurrent in other national or international populations. The other more frequent recurrent BRCA1 variant present in population databases, c. $5123 \mathrm{C}>\mathrm{A}$, has been reported in several Spanish studies [5, 38-40, $48,54,55]$, and is considered as recurrent in the Valencian community [39] and other geographical areas [13]. Haplotype analysis supports the idea that this mutation is a founder in Sephardic Jews and there may be a common origin in Sephardic Jews and the Spanish population [56]. The most recurrent BRCA2 variant in our study was c.9018C $>$ A, overrepresented in our population but not frequent in other populations $[45,46,57]$. Although the recurrent mutation c.9018C > A found in ten of our families has been reported in several populations, the databases do not consider it a frequent mutation, suggesting a possible founding effect 
in the Andalusian territory. The second most frequent recurrent mutation was c.5720_5723delCTCT, not being described a founding origin in any population at present. To test for the presence of founder effects in c.9018C > A and c.5720_5723delCTCT, we used the genotype of the polymorphic markers linked to BRCA2. All the c. $9018 \mathrm{C}>$ A families shared a common haplotype in 14 markers. All the c.5720_5723delCTCT families shared this common haplotype in 14 markers. Haplotype analysis supports the idea that this mutation has a founder origin in the south of Spain. We should emphasize that none of the BRCA $1 / 2$ recurrent mutations reported in North African studies (Algeria, Morocco, and Tunisia) were found in our study, despite the geographical proximity and the influence of the North African population on our ancestors [58].

An important finding of our study was the elevated number of novel mutations found (7.5\%). National studies analysing the mutational profile of BRCA report novel mutation rates of $2.6-6.9 \%$. This relatively high proportion of novel mutations can be considered a singularity of our study population that may be due to the specific characteristics and the lack of data available for southern Spain.

Concerning VUS, we have reported a list of variants identified in our study. Three of these had previously been reported while this is the first report for two of them, c.8022_8030delGATAATGGA and c.10186A > C. In recent years, many of the VUS that we found throughout this study have been reclassified through functional studies, co-segregation studies or coexistence with other pathogenic variants. However, we still do not know the importance of many variants. These variants are mostly missense, in frame deletion or possible splice site variants. This situation highlights the need to report BRCA data to databases and to publish research results. All the data obtained in this study have been reported to the Spanish Mutation Database [59] and international databases (ClinVar and LOVD).

The results of the study of polymorphisms and their derived haplotypes in BRCA1 and BRCA2 allowed us to establish the genetic structure of our population. The results of the haplotype frequencies in BRCA1 represented by a cladogram in our population were significantly similar to those obtained by Judkins in 55,630 patients from different populations. Some studies have reported associations between BRCA1 and BRCA2 SNPs and BC risk; however there is a lack of consistency across studies [60-63]. In our study, there was no evidence of an association between the genotypes, haplotypes and BC risk. Furthermore, no significant associations emerged between individual SNPs and molecular subtypes. Although study of SNP allele frequencies does not show strong differences between populations, it shows slight family differences with a possible founding effect.
Our study has some limitations such as the limited region studied (Andalusia), a single centre cohort (Malaga) and that other genes associated with a moderate-high risk for $\mathrm{BC}$ were not included in the analysis. On the other hand we should emphasize the large sample size (the second largest in Spain) and the homogeneous selection criteria recommended by SEOM. We should emphasize that until 2016, genetic studies regarding HBOC were practically limited to the BRCA1/BRCA2 genes, mainly due to the high cost of Sanger and MLPA sequencing techniques, but at present, most Familiar Cancer Units use gene panels related to HBOC. Since 2017 our Familiar Cancer Unit started to use a panel of 20 genes through massive sequencing that includes these and other genes related to hereditary cancer.

The BRCA1 and BRCA2 genes represent possibly the most fully sequenced genes in all human genetics. At the end of the gene-to-gene sequencing era, it is time to emphasize the importance of reporting variants and research to databases. Sharing this information is crucial for clinicians to improve patient care and allows researchers to advance in the understanding of HBOC. Currently, next-generation sequencing is providing thousands of genetic variants related to genetic diseases, and specifically HBOC. For this reason we must encourage the collection of data related to variants in BRCA1 / BRCA2 studied in the past, to investigate with more reliable information in this new era of human genetics.

\section{Conclusions}

In conclusion, this is the first study analysing BRCA1/ BRCA2 germline mutation frequencies in andalusian high risk $\mathrm{HBOC}$ patients. We report data from a large cohort of 562 high risk HBOC patients living in Malaga. We found 120 positive cases, 50 BRCA1- and 70 BRCA2-mutated patients. The most frequent mutations found in BRCA1 (c.5078_5080delCTG) and BRCA2 (c. $9018 \mathrm{C}>\mathrm{A})$ are overrepresented in our population compared to other national and international populations. Although the recurrent mutation c. $9018 \mathrm{C}>\mathrm{A}$ found in ten of our families has been reported in several studies, the databases do not consider it a frequent mutation, suggesting a possible founder effect in the Andalusian territory. We also found a relatively high proportion of novel mutations (7.5\%) and two VUS not reported in databases. In our study no evidence was detected of an association between the genotypes, haplotypes and $\mathrm{BC}$ risk and molecular subtypes.

\section{Additional files}

Additional file 1: Table S1. Index cases of women at risk for $\mathrm{HBOC}$ and Frequency of primary tumors. (DOC $22 \mathrm{~kb}$ ) 
Additional file 2: Table S2. Frequency of primary tumours in BRCA mutated families. (DOC $27 \mathrm{~kb}$ )

Additional file 3: Table S3. Breast cancer tumour phenotypes according to BRCA1 and BRCA2 mutated cases. (DOC $14 \mathrm{~kb}$ )

Additional file 4: Table S4. BRCA1 pathological germline mutations according to selection criteria and clinical characteristics. (DOC 104 kb)

Additional file 5: Table S5. BRCA2 pathological germline mutations according to selection criteria and clinical characteristics. (DOC $140 \mathrm{~kb}$ )

\section{Abbreviations}

ACMG: American College of Medical Genetics and Genomics;

AMP: Association for Molecular Pathology; BC: Breast cancer; BIC: Breast Cancer Mutation Data Base; BRCT: BRCA Terminus; CRC: Colorectal cancer; ER: Oestrogen receptor; HBOC: Hereditary breast and ovary cancer; HGMD: Human Gene Mutation Database; HGVS: Human Genome Variation Society; ICs: Index cases; IHC: Immunohistochemistry; IME: Amino acid deletion; LGRSs: Large genomic rearrangements; LOVD: Leiden Open Variation Database; LRG: Locus Reference Genomic; MLPA: Multiplex ligationdependent probe amplification; OC: Ovarian cancer; PR: Progesterone receptor; SEOM: Spanish Society of Clinical Oncology; SNP: Single nucleotide polymorphism; UMD: Universal Mutation Database; VUS: Variants of unknown significance

\section{Funding}

The funding body had no role in the design of the study and collection, analysis, and interpretation of data and in writing the manuscript.

\section{Availability of data and materials}

The datasets used and/or analysed during the current study are available from the corresponding author on reasonable request.

\section{Authors' contributions}

JP, BP, AM: Designed the study, analysed clinical and mutational data and drafted the manuscript. JMP, CFDS, DP, TV, IO: performed mutational analyses and MLPA studies. GD and IM revised clinical data. EA revised and helped to draft the manuscript. All authors approved the final manuscript.

\section{Ethics approval and consent to participate}

The study was approved by Research Ethics Committee of the University Hospital Virgen de la Victoria (Malaga). All tested individuals provided a signed informed consent following the appropriate genetic counselling.

\section{Competing interests}

The authors declare that they have no competing interests

\section{Publisher's Note}

Springer Nature remains neutral with regard to jurisdictional claims in published maps and institutional affiliations.

\section{Author details}

${ }^{1}$ Clinical Oncology Unit Hospitales Universitarios Regional y Virgen de la Victoria. Instituto de Investigación Biomédica de Málaga (IBIMA), Campus Teatinos s/n. 29010, Malaga, Spain. ${ }^{2}$ Genologica, Paseo de la Farola 16, 29016 Malaga, Spain

Received: 30 June 2017 Accepted: 21 May 2018

Published online: 08 June 2018

References

1. Ripperger T, Gadzicki D, Meindl A, Schlegelberger B. Breast cancer susceptibility: current knowledge and implications for genetic counselling. Eur J Hum Genet. 2009;17:722-31

2. Fackenthal JD, Olopade OI. Breast cancer risk associated with BRCA1 and BRCA2 in diverse populations. Nat Rev Cancer. 2007;7:937-48.

3. Brohet RM, Velthuizen ME, Hogervorst FBL, Meijers-Heijboer HEJ, Seynaeve C, Collée MJ, et al. Breast and ovarian cancer risks in a large series of clinically ascertained families with a high proportion of BRCA1 and BRCA2 Dutch founder mutations. J Med Genet. 2014;51:98-107.
4. BIC. Breast Cancer Information Core. https://research.nhgri.nih.gov/bic/. Accesed 31 Jan 2016.

5. Diez O, Gutiérrez-Enríquez S, Balmaña J. Heterogeneous prevalence of recurrent BRCA1 and BRCA2 mutations in Spain according to the geographical area: implications for genetic testing. Familial Cancer. 2010;9: 187-91.

6. Graña B, Lastra E, Llort G, Brunet J, Isla D. SEOM clinical guidelines for hereditary cancer. Clin Transl Oncol. 2011;13:580-6.

7. Untergasser A, Cutcutache I, Koressaar T, Ye J, Faircloth BC, Remm M, et al. Primer3-new capabilities and interfaces. Nucleic Acids Res. 2012;40:e115.

8. SNPCheck [Internet]. https://secure.ngrl.org.uk/SNPCheck/snpcheck.htm. Accesed 31 Jan 2016.

9. Landrum MJ, Lee JM, Benson M, Brown G, Chao C, Chitipiralla S, et al. ClinVar: public archive of interpretations of clinically relevant variants. Nucleic Acids Res. 2016:44:D862-8.

10. den Dunnen JT, Dalgleish R, Maglott DR, Hart RK, Greenblatt MS, McGowanJordan J, et al. HGVS recommendations for the description of sequence variants: 2016 update. Hum Mutat. 2016;37:564-9.

11. Richards S, Aziz N, Bale S, Bick D, Das S, Gastier-Foster J, et al. Standards and guidelines for the interpretation of sequence variants: a joint consensus recommendation of the American College of Medical Genetics and Genomics and the Association for Molecular Pathology. Genet Med. 2015;17:405-24.

12. Béroud C, Collod-Béroud G, Boileau C, Soussi T, Junien C. UMD (universal mutation database): a generic software to build and analyze locus-specific databases. Hum Mutat. 2000;15:86-94.

13. National Human Genome Research Institute. Open Access On-Line Breast Cancer Mutation Data Base. https://research.nhgri.nih.gov/bic/. Accesed 31 Jan 2016

14. Cooper DN, Stenson PD, Chuzhanova NA. The Human Gene Mutation Database (HGMD) and its exploitation in the study of mutational mechanisms. Curr. Protoc. Bioinforma. 2006:Chapter 1:Unit 1.13.

15. Fokkema IFAC, Taschner PEM, Schaafsma GCP, Celli J, Laros JFJ, den Dunnen JT. LOVD v.2.0: the next generation in gene variant databases. Hum. Mutat. 2011;32:557-63

16. Adzhubei IA, Schmidt S, Peshkin L, Ramensky VE, Gerasimova A, Bork P, et al. A method and server for predicting damaging missense mutations. Nat Methods. 2010;7:248-9.

17. Thomas PD, Kejariwal A. Coding single-nucleotide polymorphisms associated with complex vs. Mendelian disease: evolutionary evidence for differences in molecular effects. Proc Natl Acad Sci U S A. 2004;101: 15398-403.

18. Capriotti E, Calabrese R, Casadio R. Predicting the insurgence of human genetic diseases associated to single point protein mutations with support vector machines and evolutionary information. Bioinformatics. 2006;22: 2729-34.

19. Bromberg $Y$, Rost B. SNAP: predict effect of non-synonymous polymorphisms on function. Nucleic Acids Res. 2007;35:3823-35.

20. Capriotti E, Altman RB, Bromberg Y. Collective judgment predicts diseaseassociated single nucleotide variants. BMC Genomics. 2013;14(Suppl 3):S2.

21. Ng PC, Henikoff S. SIFT: predicting amino acid changes that affect protein function. Nucleic Acids Res. 2003;31:3812-4.

22. Desmet F-O, Hamroun D, Lalande M, Collod-Béroud G, Claustres M, Béroud C. Human splicing finder: an online bioinformatics tool to predict splicing signals. Nucleic Acids Res. 2009:37:e67.

23. Pertea M, Lin X, Salzberg SL. GeneSplicer: a new computational method for splice site prediction. Nucleic Acids Res. 2001;29:1185-90.

24. Sonnenburg S, Rätsch G, Jagota A, Müller KR. New Methods for Splice Site Recognition. Proc. Int. Conf. Artif. Neural Networks. 2002;

25. Eng L, Coutinho G, Nahas S, Yeo G, Tanouye R, Babaei M, et al. Nonclassical splicing mutations in the coding and noncoding regions of the ATM gene: maximum entropy estimates of splice junction strengths. Hum Mutat. 2004;23:67-76.

26. Choi Y, Sims GE, Murphy S, Miller JR, Chan AP. Predicting the functional effect of amino acid substitutions and indels. PLoS One. 2012;7:e46688.

27. Judkins T, Hendrickson BC, Deffenbaugh AM, Eliason K, Leclair B, Norton MJ, et al. Application of embryonic lethal or other obvious phenotypes to characterize the clinical significance of genetic variants found in trans with known deleterious mutations. Cancer Res. 2005:65:10096-103.

28. Rozas J, Rozas R. DnaSP version 3: an integrated program for molecular population genetics and molecular evolution analysis. Bioinformatics. 1999;15:174-5. 
29. Szabo C, Masiello A, Ryan JF, Brody LC. The breast cancer information core: database design, structure, and scope. Hum Mutat. 2000;16:123-31.

30. Caputo S, Benboudjema L, Sinilnikova O, Rouleau E, Béroud C, Lidereau R, et al. Description and analysis of genetic variants in French hereditary breast and ovarian cancer families recorded in the UMD-BRCA1/BRCA2 databases. Nucleic Acids Res. 2012:40:D992-1002.

31. Capalbo C, Ricevuto E, Vestri A, Ristori E, Sidoni T, Buffone O, et al. BRCA1 and BRCA2 genetic testing in Italian breast and/or ovarian cancer families: mutation spectrum and prevalence and analysis of mutation prediction models. Ann. Oncol. Off. J. Eur. Soc. Med. Oncol. 2006;17 Suppl 7:vii34-vii40.

32. Papi L, Putignano AL, Congregati C, Zanna I, Sera F, Morrone D, et al. Founder mutations account for the majority of BRCA1-attributable hereditary breast/ovarian cancer cases in a population from Tuscany. Central Italy Breast Cancer Res Treat. 2009;117:497-504.

33. Borg A, Haile RW, Malone KE, Capanu M, Diep A, Törngren T, et al. Characterization of BRCA1 and BRCA2 deleterious mutations and variants of unknown clinical significance in unilateral and bilateral breast cancer: the WECARE study. Hum Mutat. 2010;31:E1200-40.

34. Bonnet C, Krieger S, Vezain M, Rousselin A, Tournier I, Martins A, et al. Screening BRCA1 and BRCA2 unclassified variants for splicing mutations using reverse transcription PCR on patient RNA and an ex vivo assay based on a splicing reporter minigene. J Med Genet. 2008;45:438-46.

35. Whiley PJ, Guidugli L, Walker LC, Healey S, Thompson BA, Lakhani SR, et al. Splicing and multifactorial analysis of intronic BRCA1 and BRCA2 sequence variants identifies clinically significant splicing aberrations up to 12 nucleotides from the intron/exon boundary. Hum Mutat. 2011;32:678-87.

36. Whiley PJ, de la Hoya M, Thomassen M, Becker A, Brandão R, Pedersen IS, et al. Comparison of mRNA splicing assay protocols across multiple laboratories: recommendations for best practice in standardized clinical testing. Clin Chem. 2014;60:341-52.

37. Acedo A, Hernández-Moro C, Curiel-García Á, Díez-Gómez B, Velasco EA. Functional classification of BRCA2 DNA variants by splicing assays in a large minigene with 9 exons. Hum Mutat. 2015;36:210-21.

38. Beristain E, Martínez-Bouzas C, Guerra I, Viguera N, Moreno J, Ibañez E, et al. Differences in the frequency and distribution of BRCA1 and BRCA2 mutations in breast/ovarian cancer cases from the Basque country with respect to the Spanish population: implications for genetic counselling. Breast Cancer Res Treat. 2007;106:255-62.

39. de Juan Jl, García Casado Z, Palanca Suela S, Esteban Cardeñosa E, López Guerrero JA, Segura Huerta Á, et al. Novel and recurrent BRCA1/BRCA2 mutations in early onset and familial breast and ovarian cancer detected in the program of genetic counseling in Cancer of Valencian community (eastern Spain). Relationship of family phenotypes with mutation prevalence. Familial Cancer. 2013;12:767-77.

40. Miramar MD, Calvo MT, Rodriguez A, Antón A, Lorente F, Barrio E, et al. Genetic analysis of BRCA1 and BRCA2 in breast/ovarian cancer families from Aragon (Spain): two novel truncating mutations and a large genomic deletion in BRCA1. Breast Cancer Res Treat. 2008;112:353-8.

41. Levy-Lahad E, Friedman E. Cancer risks among BRCA1 and BRCA2 mutation carriers. Br J Cancer. 2007;96:11-5.

42. Honrado E, Benítez J, Palacios J. Histopathology of BRCA1- and BRCA2associated breast cancer. Crit Rev Oncol Hematol. 2006;59:27-39.

43. Palacios J, Robles Frias MJ, Castilla MA, Lopez-Garcia MA, Benitez J. The molecular pathology of hereditary breast Cancer. Pathobiology. 2008;75:85-94

44. Blay P, Santamaría I, Pitiot AS, Luque M, Alvarado MG, Lastra A, et al. Mutational analysis of BRCA1 and BRCA2 in hereditary breast and ovarian cancer families from Asturias (northern Spain). BMC Cancer. 2013;13:243.

45. Díez O, Osorio A, Durán M, Martinez-Ferrandis II, de la Hoya M, Salazar R, et al. Analysis of BRCA1 and BRCA2 genes in Spanish breast/ovarian cancer patients: a high proportion of mutations unique to Spain and evidence of founder effects. Hum Mutat. 2003;22:301-12

46. Esteban Cardeñosa E, Bolufer Gilabert P, Palanca Suela S, Barragán González E, Oltra Soler S, Chirivella González I, et al. BRCA1 and BRCA2 mutations in families studied in the program of genetic counselling in cancer of the Valencian community (Spain). Med. Clin. (Barc). 2008;130:121-6.

47. Macías R. Trasmisión genética del cáncer en Extremadura. Valor pronóstico del consejo genético. 2016. http://hdl.handle.net/10662/4165. Accesed 31 Dec 2016.

48. Infante M, Durán M, Esteban-Cardeñosa E, Miner C, Velasco E. High proportion of novel mutations of BRCA1 and BRCA2 in breast/ovarian cancer patients from Castilla-León (Central Spain). J Hum Genet. 2006;51:611-7

49. Vega A, Torres M, Martínez Jl, Ruiz-Ponte C, Barros F, Carracedo A Analysis of BRCA1 and BRCA2 in breast and breast/ovarian cancer families shows population substructure in the Iberian peninsula. Ann Hum Genet. 2002;66:29-36.

50. Vega A, Campos B, Bressac-De-Paillerets B, Bond PM, Janin N, Douglas FS, et al. The R71G BRCA1 is a founder Spanish mutation and leads to aberrant splicing of the transcript. Hum Mutat. 2001;17:520-1.

51. Struewing JP, Abeliovich D, Peretz T, Avishai N, Kaback MM, Collins FS, et al. The carrier frequency of the BRCA1 185delAG mutation is approximately 1 percent in Ashkenazi Jewish individuals. Nat Genet. 1995;11:198-200.

52. Roa BB, Boyd AA, Volcik K, Richards CS. Ashkenazi Jewish population frequencies for common mutations in BRCA1 and BRCA2. Nat Genet. 1996:14:185-7.

53. Judkins T, Rosenthal E, Arnell C, Burbidge LA, Geary W, Barrus T, et al. Clinical significance of large rearrangements in BRCA1 and BRCA2. Cancer. 2012;118:5210-6.

54. Salazar R, Cruz-Hernandez JJ, Sanchez-Valdivieso E, Rodriguez CA, GomezBernal A, Barco E, et al. BRCA1-2 mutations in breast cancer: identification of nine new variants of BRCA1-2 genes in a population from central western Spain. Cancer Lett. 2006:233:172-7.

55. Lovelock PK, Healey S, Au W, Sum EYM, Tesoriero A, Wong EM, et al. Genetic, functional, and histopathological evaluation of two C-terminal BRCA1 missense variants. J Med Genet. 2006;43:74-83.

56. Sagi M, Eilat A, Ben Avi L, Goldberg Y, Bercovich D, Hamburger T, et al. Two BRCA1/2 founder mutations in Jews of Sephardic origin. Familial Cancer. 2011;10:59-63.

57. Martínez-Ferrandis JI, Vega A, Chirivella I, Marín-García P, Insa A, Lluch A, et al. Mutational analysis of BRCA1 and BRCA2 in Mediterranean Spanish women with early-onset breast cancer: identification of three novel pathogenic mutations. Hum Mutat. 2003;22:417-8.

58. Cherbal F, Bakour R, Adane S, Boualga K. BRCA1 and BRCA2 germline mutation spectrum in hereditary breast/ovarian cancer families from Maghrebian countries. Breast dis. [internet], Available from. 2012:34:1-8. http://www.ncbi.nlm.nih.gov/pubmed/23697973

59. SpainMDB (Spain Mutation DataBase). http://spainmdb.isciii.es/. Accesed 31 Jan 2016

60. Sehl ME, Langer LR, Papp JC, Kwan L, Seldon JL, Arellano G, et al. Associations between single nucleotide polymorphisms in double-stranded DNA repair pathway genes and familial breast cancer. Clin Cancer Res. 2009:15:2192-203.

61. Dombernowsky SL, Weischer M, Freiberg JJ, Bojesen SE, Tybjaerg-Hansen A, Nordestgaard BG. Missense polymorphisms in BRCA1 and BRCA2 and risk of breast and ovarian cancer. Cancer Epidemiol Biomark Prev. 2009;18:2339-42.

62. Juwle A, Saranath D. BRCA1/BRCA2 gene mutations/SNPs and BRCA1 haplotypes in early-onset breast cancer patients of Indian ethnicity. Med Oncol. 2012;29:3272-81.

63. Freedman ML, Penney KL, Stram DO, Riley S, McKean-Cowdin R, Le Marchand L, et al. A haplotype-based case-control study of BRCA1 and sporadic breast cancer risk. Cancer Res. 2005;65:7516-22.

\section{Ready to submit your research? Choose BMC and benefit from:}

- fast, convenient online submission

- thorough peer review by experienced researchers in your field

- rapid publication on acceptance

- support for research data, including large and complex data types

- gold Open Access which fosters wider collaboration and increased citations

- maximum visibility for your research: over $100 \mathrm{M}$ website views per year

At BMC, research is always in progress.

Learn more biomedcentral.com/submissions 